\title{
The therapeutic effect of mesenchymal stem cells on pulmonary myeloid cells following neonatal hyperoxic lung injury in mice
}

\author{
Ali Al-Rubaie', Andrea F. Wise', Foula Sozo', Robert De Matteo', Chrishan S. Samuel², Richard Harding ${ }^{1}$
} and Sharon D. Ricardo ${ }^{1 *}$ (D)

\begin{abstract}
Background: Exposure to high levels of oxygen (hyperoxia) after birth leads to lung injury. Our aims were to investigate the modulation of myeloid cell sub-populations and the reduction of fibrosis in the lungs following administration of human mesenchymal stem cells (hMSC) to neonatal mice exposed to hyperoxia.
\end{abstract}

Method: Newborn mice were exposed to $90 \% \mathrm{O}_{2}$ (hyperoxia) or $21 \% \mathrm{O}_{2}$ (normoxia) from postnatal days $0-4$. A sub-group of hyperoxia mice were injected intratracheally with $2.5 \times 10^{5} \mathrm{hMSC}$. Using flow cytometry we assessed pulmonary immune cells at postnatal days 0, 4,7 and 14. The following markers were chosen to identify these cells: $\mathrm{CD}^{+} 5^{+}$(leukocytes), Ly6C $\mathrm{Ly}^{+} \mathrm{G}^{+}$(granulocytes), $\mathrm{CD} 11 \mathrm{~b}^{+} \mathrm{CD} 11 \mathrm{c}^{+}$(macrophages); macrophage polarisation was assessed by F4/80 and CD206 expression. hMSCs expressing enhanced green fluorescent protein (eGFP) and firefly luciferase (fluc) were administered via the trachea at day 4. Lung macrophages in all groups were profiled using next generation sequencing (NGS) to assess alterations in macrophage phenotype. Pulmonary collagen deposition and morphometry were assessed at days 14 and 56 respectively.

Results: At day 4, hyperoxia increased the number of pulmonary Ly6C $C^{+} L y 6 G^{+}$granulocytes and F4/80 ${ }^{\text {low }} \mathrm{CD} 206^{\text {low }}$ macrophages but decreased $\mathrm{F} 4 / 80^{\text {high }} \mathrm{CD} 206^{\text {high }}$ macrophages. At days 7 and 14 , hyperoxia increased numbers of $\mathrm{CD}_{4} 5^{+}$leukocytes, $\mathrm{CD} 11 \mathrm{~b}^{+} \mathrm{CD} 11 \mathrm{c}^{+}$alveolar macrophages and $\mathrm{F} 4 / 80^{\text {low }} \mathrm{CD} 206^{\text {low }}$ macrophages but decreased F4/80 ${ }^{\text {high }}$ CD206 $6^{\text {high }}$ macrophages. hMSCs administration ameliorated these effects of hyperoxia, notably reducing numbers of $\mathrm{CD} 11 \mathrm{~b}^{+} \mathrm{CD} 11 \mathrm{c}^{+}$and $\mathrm{F} 4 / 80^{\text {low }} \mathrm{CD} 206^{\text {low }}$ macrophages; in contrast, $\mathrm{F} 4 / 80^{\text {high }} \mathrm{CD} 206^{\text {high }}$ macrophages were increased. Genes characteristic of anti-inflammatory 'M2' macrophages (Arg1, Stat6, Retnla, Mrc1, II27ra, Chil3, and II12b) were up-regulated, and pro-inflammatory 'M1' macrophages (Cd86, Stat1, Socs3, Slamf1, Tnf, Fcgr1, II $26,\|6\|$,16 , and $1127 \mathrm{ra}$ ) were downregulated in isolated lung macrophages from hyperoxia-exposed mice administered hMSCs, compared to mice without hMSCs. Hydroxyproline assay at day 14 showed that the 2-fold increase in lung collagen following hyperoxia was reduced to control levels in mice administered hMSCs. By day 56 (early adulthood), hMSC administration had attenuated structural changes in hyperoxia-exposed lungs.

Conclusions: Our findings suggest that hMSCs reduce neonatal lung injury caused by hyperoxia by modulation of macrophage phenotype. Not only did our cell-based therapy using hMSC induce structural repair, it limited the progression of pulmonary fibrosis.

Keywords: Alveolar macrophages, Neonatal hyperoxia, Mesenchymal stem cells

\footnotetext{
* Correspondence: Sharon.ricardo@monash.edu

'Department of Anatomy and Developmental Biology, Biomedicine

Discovery Institute, Monash University, Clayton, VIC 3800, Australia

Full list of author information is available at the end of the article
}

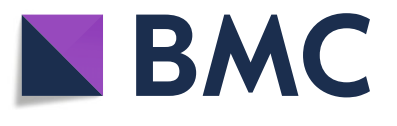

(c) The Author(s). 2018 Open Access This article is distributed under the terms of the Creative Commons Attribution 4.0 International License (http://creativecommons.org/licenses/by/4.0/), which permits unrestricted use, distribution, and reproduction in any medium, provided you give appropriate credit to the original author(s) and the source, provide a link to the Creative Commons license, and indicate if changes were made. The Creative Commons Public Domain Dedication waiver (http://creativecommons.org/publicdomain/zero/1.0/) applies to the data made available in this article, unless otherwise stated. 


\section{Background}

Preterm infants experience a range of debilitating health issues primarily resulting from lung immaturity, and few treatment options are available [1-3]. Owing to lung underdevelopment, preterm infants often require mechanical ventilation with hyperoxic gas in order to survive $[4,5]$. However, high levels of oxygen or prolonged use of ventilators can damage the lungs and interrupt normal alveolar and bronchiolar development, which may lead to chronic lung diseases known as bronchopulmonary dysplasia (BPD) [6]. It is common, that preterm infants that are born at less than 32 weeks of gestation have increased progression of other short and long-term respiratory illnesses, such as asthma and chronic obstructive pulmonary disease (COPD) [7].

Experimental studies have attempted to reduce the negative effects of hyperoxia on the developing lungs; however, clinical translation has been disappointing to date. Consequently, there is still no effective treatment for BPD in preterm infants [8-10]. BPD is associated with the inflammation of lungs, which typically involves the recruitment of monocytes that differentiate into alveolar macrophages [11]. Macrophages are a heterogeneous cell type that can be broadly categorised into two groups: "classically activated" M1 macrophages that have pro-inflammatory functions and M2 macrophages are "alternatively activated" cells that play a reparative or regulatory role [12-14].

Human mesenchymal stem cells (hMSCs) have the ability to alter macrophage phenotype from an inflammatory to anti-inflammatory phenotype that may be therapeutically beneficial to treat injured lungs [15]. There have been completed or ongoing clinical trials involving cell therapies that delay the progression or reverse a variety of immune and non-immune diseases in the lung and other organs [16]. Preclinical and clinical data support the use of cell therapy to treat COPD, acute adult lung injury and severe chronic asthma [17]. Recently, the protective effects of stem cells in lung diseases, for example BPD, have been demonstrated [18]. Studies have shown that stem cells protect the newborn injured lung from BPD and aid in endogenous repair of the injured lung tissue, either by differentiation into lung parenchymal cells [19], or into type II alveolar epithelial cells [20], via the secretion of factors [21]. However, some limitations to the studies have also been reported such as risks associated with possible immune reactions against hMSCs [22]. Understanding the therapeutic benefit of hMSCs in neonatal hyperoxic lung injury in mice remaining largely unclear, specifically, the ability to alter the lung immune cells population/s.

This study aimed to determine the effectiveness of human bone marrow-derived hMSC therapy on neonatal hyperoxia, through the modulation of pulmonary immune cells and lung injury-induced collagen deposition in mice, based on the alteration of macrophage phenotype. We showed that hMSCs tracked to the injured lung following intratracheal injection where they modulated macrophage phenotype leading to reduced collagen deposition, thus improving lung structure.

\section{Methods \\ Experimental animals}

This study was performed using pups at embryonic day 14 timed-pregnant C57BL/6 J mice obtained from the Monash Animal Research Platform (MARP), housed at Monash University, Clayton. The study was conducted under animal ethics number MARP2014/092 issued by Monash Animal Ethics Committee. Pups were born at term, and the day designated as postnatal day 0 . The exposure of lungs to $90 \% \mathrm{O}_{2}$ was used to induce injury and the effectiveness of hMSC treatment on hyperoxiainduced lung injury was assessed. Newborn mice were exposed to either normoxia $21 \% \mathrm{O}_{2}$ (control group) or $90 \% \mathrm{O}_{2}$ (hyperoxia) from postnatal day 0 to day 4 . The oxygen concentration was maintained and monitored throughout the period of hyperoxia dams were rotated with non-hyperoxia dams (SWAP dams) every $24 \mathrm{~h}$. The mice received standard chow ad libitum under $12 / 12 \mathrm{~h}$ day/ night cycle. At day 4, sub-groups within the hyperoxia groups were injected directly into the trachea with hMSCs purchased from the Tulane Centre for Stem Cell Research and Regenerative Medicine (Tulane University, New Orleans, LA) [23] at passage four. $2.5 \times 10^{5} \mathrm{hMSCs}$ were suspended in $10 \mu \mathrm{lBS}$ and delivered via intratracheal injection using a $1 \mathrm{ml}$ insulin syringe and a $29 \mathrm{G}$ hypodermic needle. Mouse lungs were collected at days 0, 4, 7 and 14 .

\section{Bioluminescence imaging}

For cell tracing of hMSCs in vivo, sub-groups of hyperoxia-exposed mice $(n=8)$ were administered $2.5 \times 10^{5}$ hMSCs expressing enhanced green fluorescent protein (eGFP) and firefly luciferase (fluc) in $10 \mu \mathrm{l}$ PBS at postnatal day 4 [23]. To image the hMSCs in vivo following delivery, anesthetized mice were injected intraperitoneally with $200 \mu \mathrm{l}$ of D-luciferin $(15 \mathrm{mg} / \mathrm{ml}$ in PBS; VivoGlo Luciferin, Promega, San Luis Obispo, CA, USA) and imaged on days 0,1 and 3 following cell delivery using a IVIS 200 system (Xenogen, Alameda, CA, USA). The fluc luminescent signal was captured and analysed as photons $/ \mathrm{sec} / \mathrm{cm}^{2}$ (Living Image 3.2, Xenogen).

\section{Lung immune cells preparation}

Preparation of lung tissue Freshly excised lungs were kept in cold FACS -fluorescence-activated cell sortingbuffer (Phosphate-buffered saline accompanied with $0.2 \%$ Bovine serum albumin, $0.02 \%$ Sodium azide and $5 \mathrm{mM}$ EDTA). With surgical scissors, the main airways were removed and the lungs were cut into small portions before 
enzymatic digestion, for $45 \mathrm{~min}$, in $1 \mathrm{~mL}$ of dissociation media consisting of HBSS- Hanks' Balanced Salt Solution- (Sigma-Aldrich, St. Louis, USA) containing $3 \mathrm{mg} / \mathrm{mL}$ collagenase/dispase (Roche Applied Science, Penzberg, Germany) and $0.2 \mathrm{mg} / \mathrm{mL}$ DNase type 1 (Roche Applied Science). To eliminate red blood cells, the lung single cell suspensions were incubated in $1 \mathrm{~mL}$ of red blood cell lysis buffer $(8.3 \mathrm{~g} / \mathrm{L} \mathrm{NH} 4 \mathrm{Cl}, 10 \mathrm{mM}$ Tris- $\mathrm{HCl}$, and $\mathrm{pH}$ 7.5) for $1 \mathrm{~min}$. Samples were filtered through a $100 \mu \mathrm{m}$ nylon cell strainer (BD Bioscience, San Jose, USA) before markers labelling.

Flow-cytometry Cells counts were performed using a Z2 Coulter Counter (Beckman Coulter, USA). $3 \times 10^{6}$ cells from single cell suspensions were incubated at $4{ }^{\circ} \mathrm{C}$ for $20 \mathrm{~min}$ with the following fluorochrome-conjugated anti-mouse markers: anti-CD45 APC-Cy7 (clone 30-F11; Biolegend, San Diego, USA), anti-CD11b PE-Cy7 (clone M1/70; BD Biosciences), anti-CD11c Pacific Blue (clone N418; Biolegend), anti-Ly6C FITC (clone HK1.4; Biolegend), anti-Ly6G Alexa Fluor 647 (clone 1A8; Biolegend), anti-F4/ 80 APC (clone BM8; eBioscience), and anti-CD206 (mannose receptor; MR) Alexa Fluor 488 (clone C068C2; Biolegend). Fc receptor block (anti-CD16/32 antibody) was added to all markers cocktails. Intracellular CD206 labelling was performed using a CytoFix/CytoPerm kit (BD Biosciences, USA). After surface receptor labelling, cells were permeabilized and incubated with the marker for $30 \mathrm{~min}$ at $4{ }^{\circ} \mathrm{C}$ in the dark before being washed twice in $1 \times$ Perm/Wash buffer (BD Biosciences) and resuspend in FACS buffer. A BD FACS Canto II flow cytometer (BD Biosciences) was used to acquire data. Data was analysed using FlowLogic FCS analysis software (Inivai Technologies, Melbourne, Australia).

Alveolar macrophages total-RNA sequencing At postnatal day14, $1 \mathrm{X} 10^{5} \mathrm{CD} 45^{+} \mathrm{CD} 11 \mathrm{~b}^{+} \mathrm{CD} 11 \mathrm{c}^{+}$alveolar macrophages were sorted from mouse lungs (total $n=9 ; 3$ from each treatment group) via an influx sorter (Flowcore, Monash University, Victoria, Australia). Total RNA was extracted from the sorted macrophages using RNeasy Kit following the manufacturer's protocol (Qiagen, Hilden, Germany). The integrity of the samples was measured using the Agilent Bioanalyzer 2100 with B.02.08.SI648 (SR3) software and a microfluidics device, in conjunction the associated hardware and chemistry (Agilent Technologies, Waldbronn, Germany). Samples were used to construct Illumina sequencing libraries using the following Takara SMART-seq Ultra Low Input version 4 according to the manufacturer's instructions (Clontech Takara SMART-Seq V4 Ultra Low Input RNA Kit for Sequencing User Manual v.01251). Libraries were quantitated using a Qubit DNA HS kit, which incorporates a doublestranded DNA-specific fluorescent dye (Invitrogen, Carlsbad
CA., USA). Libraries were sized and checked for adapter contamination using the Agilent Bioanalyzer 2100 microfluidics device, in conjunction with Agilent DNA HS kits and chemistry (Agilent Technologies, Waldbronn, Germany). These libraries were sequenced using the following chemistry and conditions in NextSeq500 - High-Output SBS version 2 (Illumina 15,046,563 v02), with library concentration $1.8 \mathrm{pM}$ and reading length $1 \mathrm{X} 75 \mathrm{~b}$ according to the manufacturer's instructions. The differential gene expression was performed with the voom [24] limma package (v3.34.1) [25].

\section{Lung fibrosis profile and structural changes}

Hydroxyproline assay On day 14, lung tissue from mouse pups $(n=8)$ were assessed for total collagen content, determined by hydroxyproline content as previously described [23] . Hydroxyproline values were then converted to collagen content by multiplying by a factor of 6.94 as hydroxyproline represents; $14.4 \%$ of the amino acid composition of collagen in most mammalian tissues [26]. This was determined using a standard curve of purified trans-4-hydroxy-L-proline (Sigma-Aldrich) and further expressed as a percentage of the dry tissue weight to yield collagen concentration.

Morphometric lung analysis At day 56, lung tissue sections from mouse ( $n=8 /$ group) were randomly selected from the left lung were embedded in paraffin for histological staining. Paraffin-embedded sections of lung tissue were stained with picrosirius red. Sections $5 \mu \mathrm{m}$ thick were examined by light microscopy (X200 magnification) and five random sections were captured using a digital camera (SPOT Insight 4Meg Fire Wire Color Mosaic 14.2, Diagnostic Instruments, USA). To assess lung injury, mean linear intercept (MLI) was measured using software ImageJ, version 1.47 (Wayne Rasband, NIH, Bethesda, MD, USA). Large airways and vessels were avoided. The program placed horizontal lines, $30 \mu \mathrm{m}$ apart, across each section of lung. Then the number of times the lines intercepted the alveoli (intercepts) was calculated. All counting methods have been described previously [27].

\section{Statistical analysis}

Data were analysed using GraphPad Prism software version 7.0c (GraphPad Software Inc., San Diego, USA) and IBM SPSS statistics (The Apache Software Foundation). A $t$-test (unpaired, two-tailed) was used to analyse differences between the normoxia and hyperoxia groups at day 4. A one-way analysis of variance with a Tukey's multiple comparisons test was used to analyse data contained in the normoxia, hyperoxia and hyperoxia + hMSC groups at day 7 and day 14 . Data are given as means \pm SEM and $P<0.05$ was considered statistically significant. 


\section{Results}

\section{Bioluminescence imaging of hMSCs}

Following intratracheal injection of hMSCs at day 4, whole body bioluminescence imaging was used to confirm the fate of the hMSCs in vivo. The intensity of bioluminescence has previously been shown to be directly proportional to the number of labelled hMSCs residing in the host tissue [28]. hMSCs administered to hyperoxia and normoxia exposed lungs were found to localize in the lungs when assessed at days 0 and 1 post-administration (Fig. 1). hMSCs were clearly detected in the thoracic area of the pups, corresponding to the position of the lungs. Bioluminescence detection of hMSCs reduced over time, whereby there was no signal by day 3 .

\section{Flow cytometry}

Flow cytometry was performed on days $0,4,7$ and 14 to evaluate the effect of hyperoxia and hMSC treatment on the numbers of lung $\mathrm{CD} 45^{+}$leukocytes and sub-populations of myeloid cells. Fig. 2 shows the gating strategy used for analysing immune cells in the lung. Immunofluorescent staining of the mononuclear cells using markers cocktail was used to identify the $\mathrm{CD} 45^{+}$leukocytes.

There were significant changes in the $\mathrm{CD} 45^{+}$leukocytes populations and the sub-populations including granulocytes populations expressing $\mathrm{Ly}_{6} \mathrm{C}^{+} \mathrm{Ly} 6 \mathrm{G}^{+}$, alveolar macrophages populations expressing $\mathrm{CD} 11 \mathrm{~b}^{+} \mathrm{CD} 11 \mathrm{c}^{+}$and their sub-populations macrophages phenotypes $\mathrm{F} 4 / 80^{\text {low }} \mathrm{CD} 206^{\text {low }}$ ' $\mathrm{M} 1$ ' and $\mathrm{F} 4 / 80^{\text {high }} \mathrm{CD} 206^{\text {high }}$ 'M1'among the three groups analysed.

\section{CD45 ${ }^{+}$leukocytes}

At day 7 and day 14 there was a greater number of $C D 45^{+}$ cells $(P<0.05)$ in hyperoxia-exposed lungs compared to normoxia-exposed lungs (Fig. 3). Importantly, at day 7 hMSC administration to mice exposed to hyperoxia significantly reduced the number of $\mathrm{CD} 45^{+}$cells in the lungs compared to hyperoxia-exposed lungs $(P<0.05)$.

\section{Granulocytes}

At day 4 , the number of $\mathrm{Ly} 6 \mathrm{C}^{+} \mathrm{Ly} 6 \mathrm{G}^{+}$granulocytes were significantly higher $(P<0.05)$ in lungs from hyperoxiaexposed mice, compared to mice receiving normoxia. Interestingly, the administration of MSCs to mice with lungs exposed to hyperoxia had no impact on the numbers of granulocytes at day 7 and 14 (Fig. 4).

\section{Alveolar macrophages}

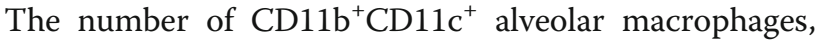
representing a sub-population of the $\mathrm{CD} 45^{+}$cells. At day 4 , there are no significant differences between the treatment groups. However, by day 7 and 14 there were significantly more $\mathrm{CD} 11 \mathrm{~b}^{+} \mathrm{CD} 11 \mathrm{c}^{+}$macrophages in the lungs from mice exposed to hyperoxia compared to normoxia $(P<0.05)$. In comparison, hMSC administration starting at day 4 was effective in reducing the number of alveolar macrophages at day 7 and 14, comparable to the normoxia group $\left(P<0.05\right.$; Fig. 5). From the $\mathrm{CD} 11 \mathrm{~b}^{+} \mathrm{CD} 11 \mathrm{c}^{+}$alveolar macrophages, two sub-populations - phenotypes- were examined using F4/80 and CD206 expression:
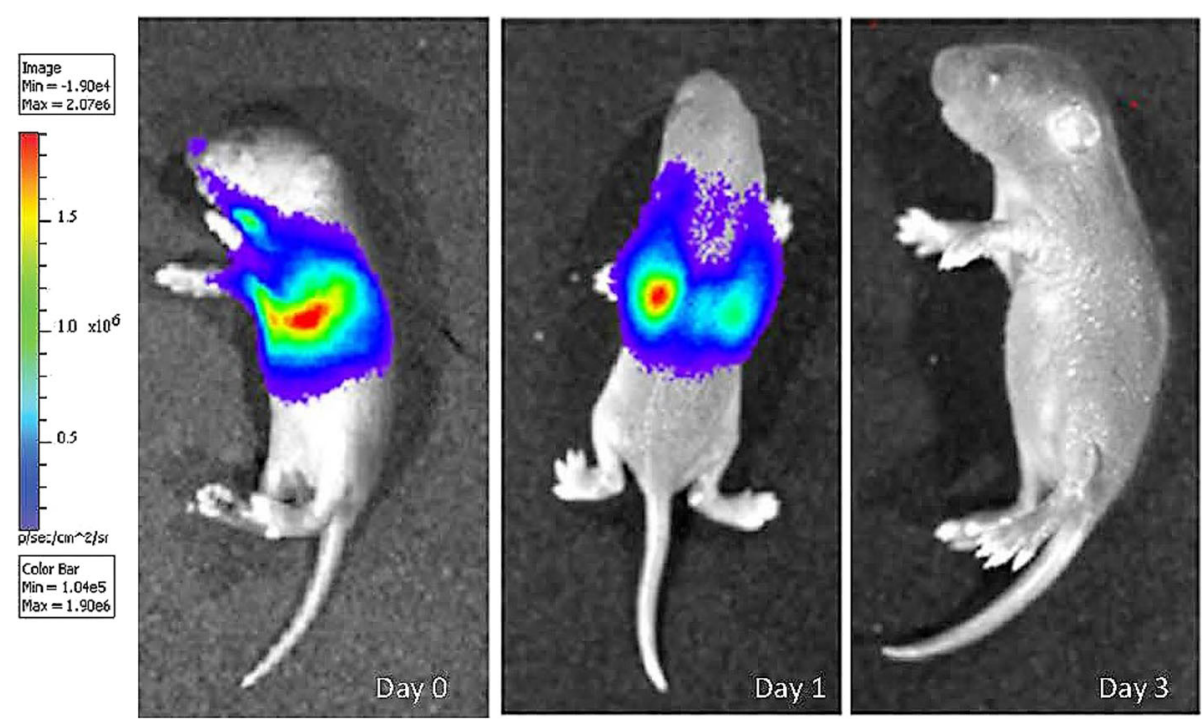

Fig. 1 Luciferin/GFP +MSCs were injected into mice after hyperoxia $n=8$, and the bioluminescence fluc signal was examined at time 0 and day 1 and 3 after luciferase injection. Bioluminescence imaging showing the intensity of fluc signal representing MSC localization to lungs. Red signals indicate high intensity of MSCs and blue indicates low intensity - see heatmap panel 


\section{a Population Hierarchy in hyperoxic lungs} Total Acquisition

- 'Single' Cells (FSC-A vs. FSC-H) (b)

- 'Non-debris' cells (FSC-A vs. SSC-A) (b)

- Leukocytes (FSC-A vs. CD45') (b)

- Alveolar Macrophages (CD11 b vs. CD11 $\left.\mathrm{c}^{+}\right)$(c)

- M1 M2 Macrophages (F4/80+ vs. CD206 ${ }^{+}$) (d)

- Granulocytes (Ly6C $C^{+}$vs. Ly6G ${ }^{+}$) (e)

b

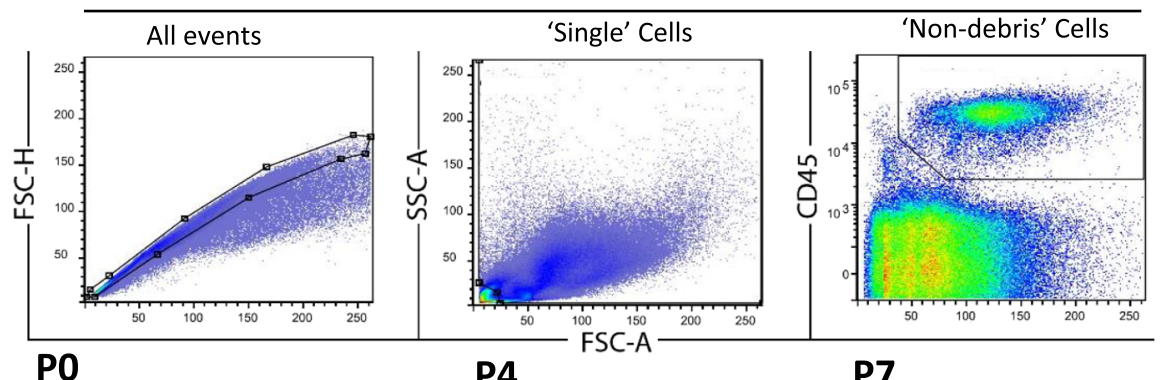

C

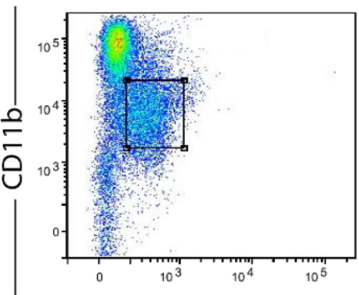

P4

P7

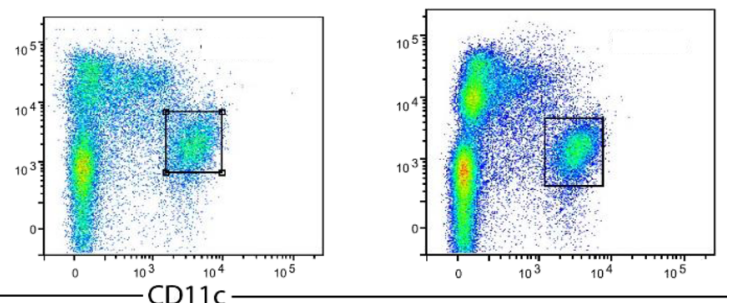

d

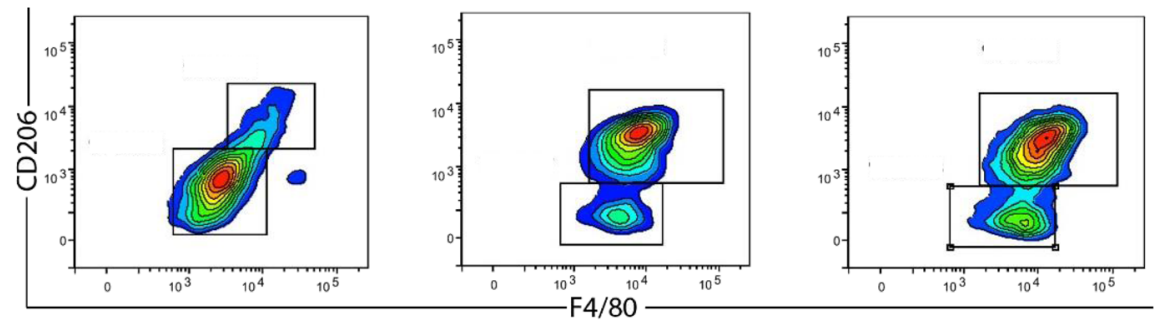

e

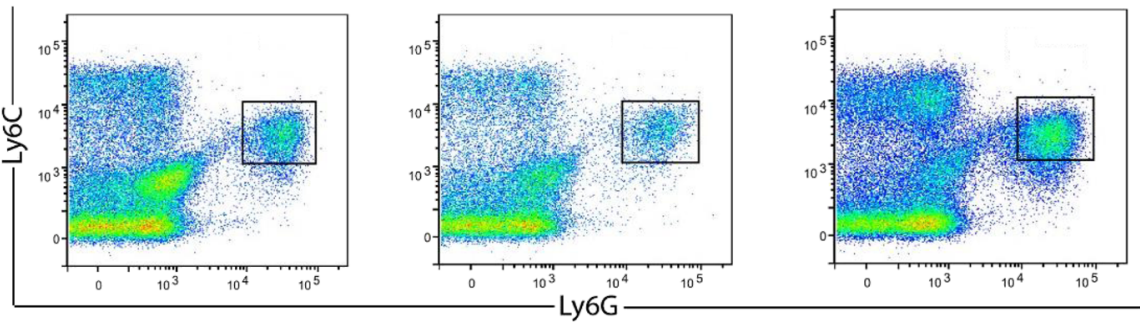

Fig. 2 The representative gating strategy for assessing myeloid cell sub-populations in the lung. The population hierarchy shows the gating strategy (a). 'Single' cells (excluding doublets and triplets) were selected with a polygon gate on a FSC-A vs. FSC-H dot plot (b). Single cells were gated on the resulting daughter population on a FSC-A vs. SCA-A dot plot (b). A 'Live' cell gate (which excludes debris) was created with the aid of the CD45 cells on FSC-A vs. $C D 45^{+}$(b). $C D 45^{+} \mathrm{CD} 11 \mathrm{~b}^{+} \mathrm{CD} 11 \mathrm{c}^{+}$alveolar macrophages were selected with a gate (b), $\mathrm{CD} 45^{+} \mathrm{F} 4 / 80^{+} \mathrm{CD} 206^{+} \mathrm{M} 1$ vs. M2 macrophages were selected with a gate $(\mathbf{d})$ and $\mathrm{CD}_{4} 5^{+} \mathrm{Ly} 6 \mathrm{C}^{+} \mathrm{Ly} 6 \mathrm{G}^{+}$granulocytes (e) were selected for further analysis of myeloid cell subsets. Plots in $\mathrm{c}$, $\mathrm{d}$ and e are from a hyperoxic lung taken at day 0 , day 4 , and day 7 from left to right

\section{- M1 macrophages}

The number of $\mathrm{F} 4 / 80^{\text {low }} \mathrm{CD} 206^{\text {low }}$ macrophages subpopulation, that represent an inflammatory 'M1' phenotype, significantly changed among the treatment groups. Hyperoxia caused a significant rise in the number of $\mathrm{F} 4 / 80^{\text {low }} \mathrm{CD} 206^{\text {low }}$ macrophages at days 4,7 and 14, compared to the normoxia group $(P<0.05$; Fig. 6). This increase in cell number at day 


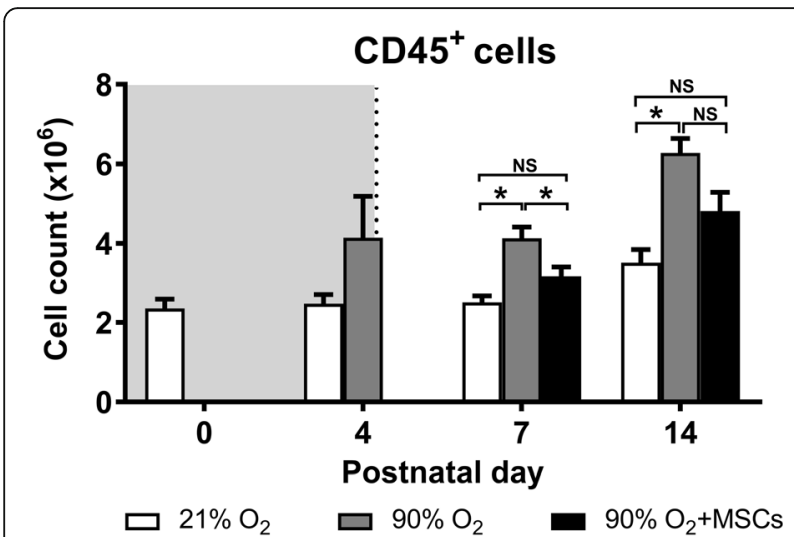

Fig. $3 \mathrm{CD} 5^{+}$myeloid cell count in lungs from mice following normoxia ( $\square$, 90\% $\mathrm{O}_{2}\left(\square\right.$ ) and $90 \% \mathrm{O}_{2}$ with administration of hMSC $\square$ delivered at day 4. The shaded area (grey) depicts period of hyperoxia exposure (postnatal days $0-4$ ) prior to delivery of hMSCs (dotted line) on day 4. For all treatment groups, $n=8$ per group. Data are shown as mean \pm SEM. ${ }^{*}=p<0.05$

14 was attenuated following the hMSC treatment, where there was no significant difference between normoxia and hMSC injected hyperoxia groups.

\section{- M2 macrophages}

The number of F4/80 ${ }^{\text {high }} \mathrm{CD} 206^{\text {high }}$ cells, that represent an anti-inflammatory 'M2' phenotype, decreased in mice exposed to hyperoxia compared to normoxia over all time points studied $(P<0.05$; Fig. 7). At day 4, hyperoxia exposure resulted in a significant decrease in the number of F4/80 highCD206 ${ }^{\text {high }}$ cells in mouse lungs compared to the normoxia group $(P<0.05)$. At day 7 and 14 , hMSC

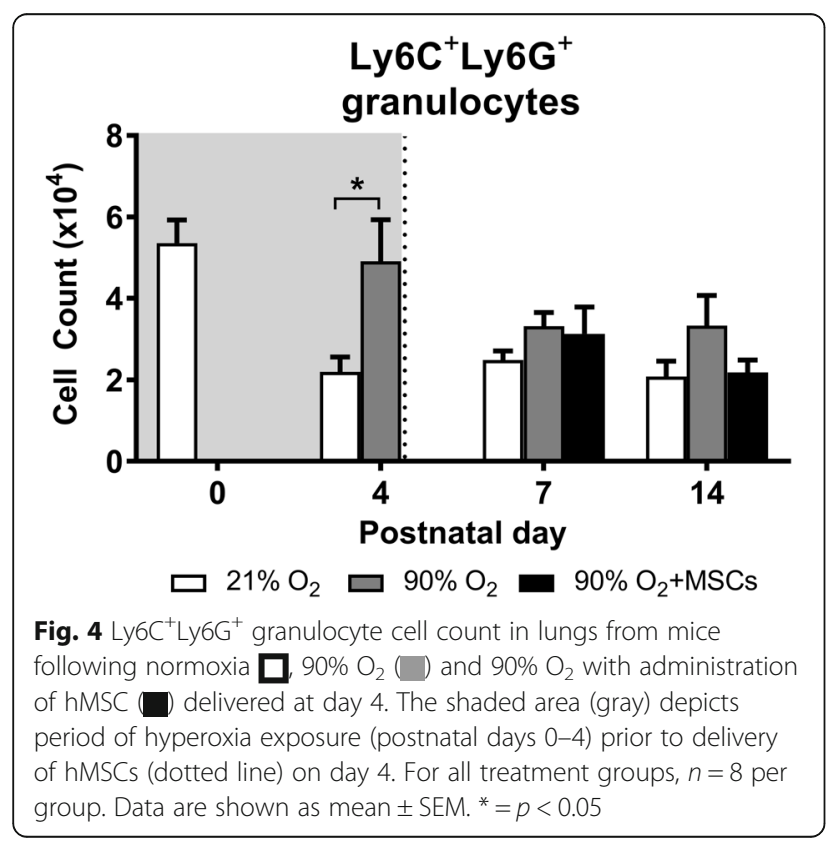

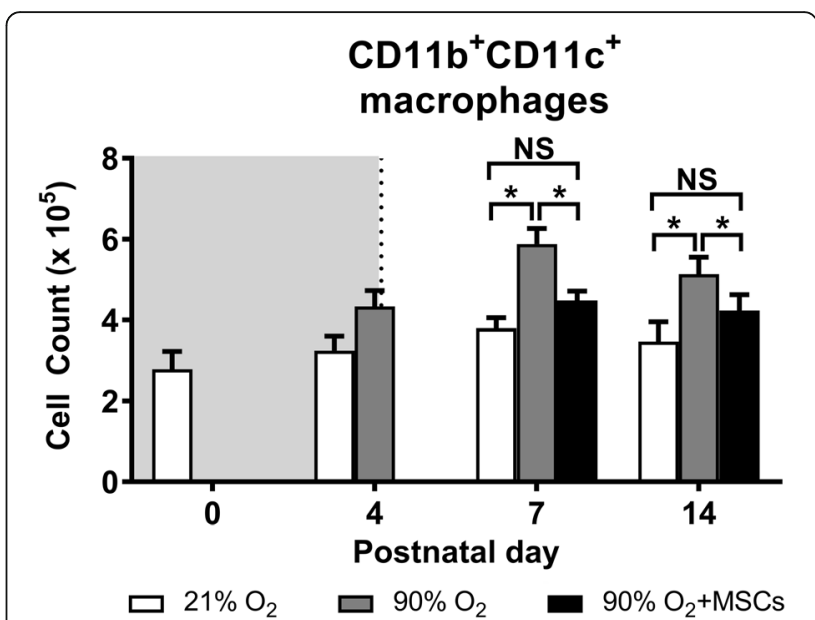

Fig. $5 \mathrm{CD} 11 \mathrm{~b}^{+} \mathrm{CD} 11 \mathrm{c}^{+}$macrophage cell count in lungs from mice following normoxia $(\square), 90 \% \mathrm{O}_{2}(\square)$ and $90 \% \mathrm{O}_{2}$ with administration of hMSC $\square$ delivered at day 4. The shaded area (gray) depicts period of hyperoxia exposure (postnatal days 0-4) prior to delivery of hMSCs (dotted line) on day 4 . For all treatment groups, $n=8$ per group. Data are shown as mean \pm SEM. ${ }^{*}=p<0.05$ and NS $=$ not significant

administration was able to effectively increase $(P<0.05)$ the number of $\mathrm{F} 4 / 80^{\text {high }} \mathrm{CD} 206^{\text {high }} \mathrm{M} 2$ macrophages. At day 14, the cell number was restored to control levels where there was no significant difference between normoxia and hMSC injected hyperoxia groups.

Alveolar macrophages total- RNA sequencing Using next generation sequencing, macrophage total-RNA gene expression was analysed assessing specific genes

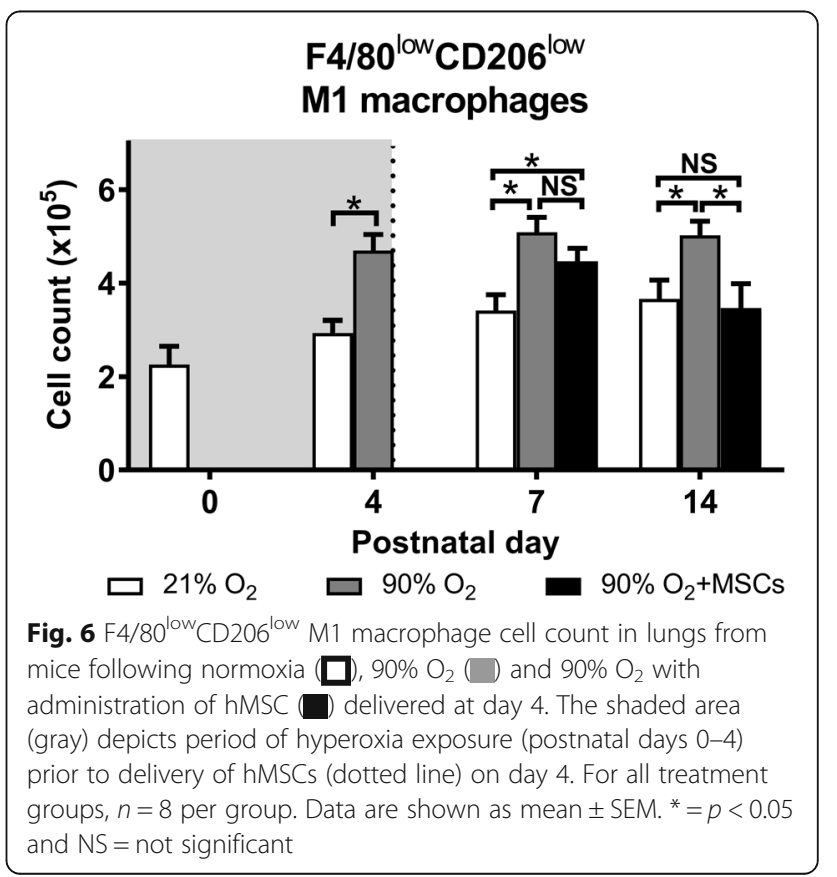




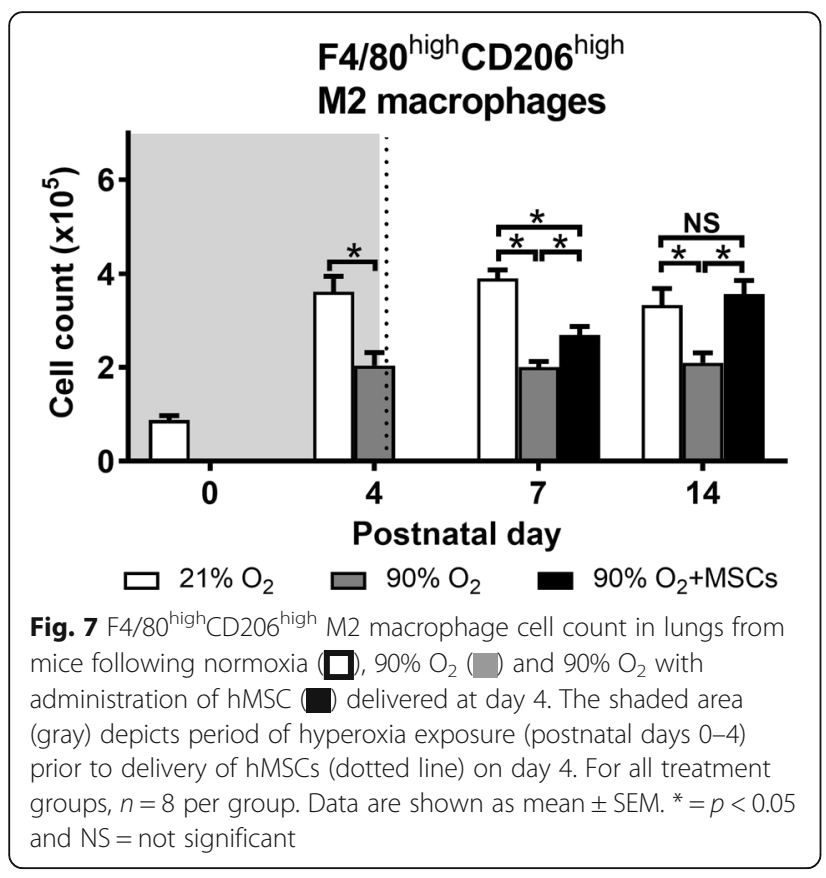

characteristic of M1- proinflammatory macrophages including Cd86, Stat1, Socs3, Slamf1, Tnf, Fcgr1, Il12b, Il6, $I l 1 b$, and Il27ra. These genes were up-regulated in isolated lung macrophages from mice exposed to hyperoxia compared to mice receiving hMSCs. Furthermore, genes related to M2- anti-inflammatory macrophages showed overall down regulation in macrophages isolated from lungs of hyperoxic mice receiving hMSCs. These genes included Arg1, Stat6, Retnla, Mrc1, Il27ra, Chil3, and Il12b [29], as shown in Additional file 1: Table S1.

\section{Collagen accumulation is decreased by hMSCs in hyperoxic mouse lungs}

Hydroxyproline analysis of total collagen concentration was used to assess the progression of lung fibrosis in mouse lungs exposed to either normoxia or hyperoxia in comparison to mice exposed to hyperoxia under the treatment of hMSCs (Fig. 8). Mice exposed to hyperoxia showed a 2 fold increase in lung collagen accumulation (percent collagen content per dry weight tissue) at day 14 compared with the normoxia animals. There was also a significant reduction in total collagen content, representing reduced interstitial fibrosis, in lungs from hMSCtreated mice exposed to hyperoxia. The collagen content in these mice was comparable to control mice.

\section{Lung structure improves in hyperoxic mice following hMSC delivery}

The assessment of lung histology showed that the administration of hMSC to mice exposed to hyperoxia improved lung architecture and alveolar structure (Fig. 9b), which was consistent with an increased MLI (Fig. 9a). At

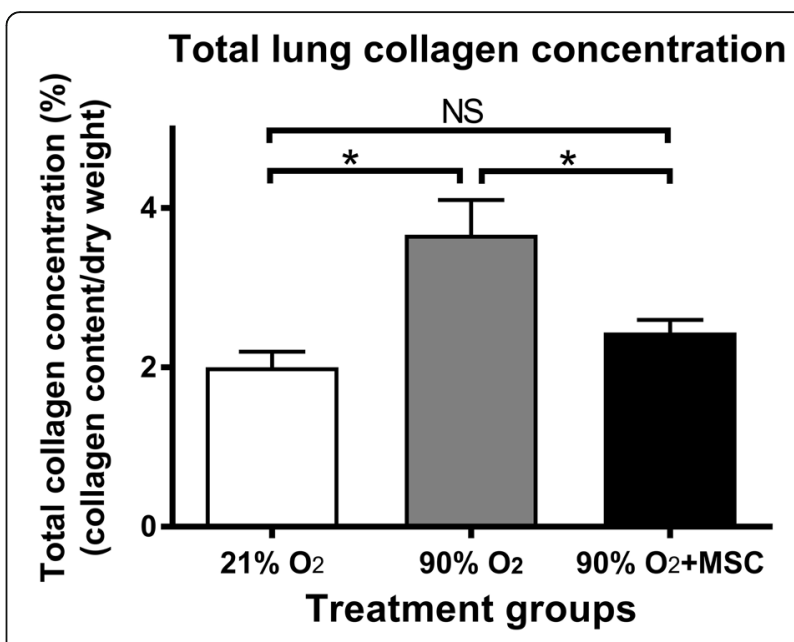

Fig. 8 hMSCs reduce collagen accumulation in the lung following hyperoxic injury total lung collagen concentration (\% collagen content/dry weight tissue) in normoxia, $90 \% \mathrm{O}_{2}$ and $90 \% \mathrm{O}_{2}$ with administration of hMSC on postnatal day 14. For all treatment groups $n=8$. Data are shown as mean \pm SEM. ${ }^{*}=p<0.05$ and $\mathrm{NS}=$ not significant

day 56, MLI was higher in hyperoxia treated mouse lungs $41.84 \pm 0.39 \mu \mathrm{m}$ ) compared to the lungs of mice exposed to normoxia $(29.74 \pm 0.48 \mu \mathrm{m})$. However, the hyperoxia+hMSC group showed a noticeable amelioration of injury as evidenced by a decrease in the MLI compared to hyperoxia without administration of hMSC $(31.27 \pm 0.56 \mu \mathrm{m}$ vs. $41.84 \pm 0.39 \mu \mathrm{m} ; P<0.05)$. The MLI from lungs of normoxic mice was not significantly different from the MLI of mice exposed to hyperoxia administered MSCs.

\section{Discussion}

The administration of hMSCs to the neonatal lung ameliorated the hyperoxia-induced injuries, including reducing collagen deposition. Additionally, the hMSC administration was found to effectively reduce the hyperoxia-induced infiltration and phenotype of subpopulations of macrophages into the damaged lung.

To study the effects of hMSC therapy in the neonatal lung, a mouse model was used which mimics the effects of neonatal lung hyperoxia in human preterm babies [1]. This model has provided significant insights into the lung pathology induced by exposing the developing lungs to hyperoxic gas [30]. This study provides the first evidence that a non-surgical, intra-airway route of administration in mice can effectively deliver hMSCs to the neonatal lung as early as one-hour post-injection where they remained elevated for $24 \mathrm{~h}$. Confirmation of MSCs in damaged lungs has been difficult to ascertain due to the entrapment of hMSCs in lung capillaries when delivered intravenously [31]. 


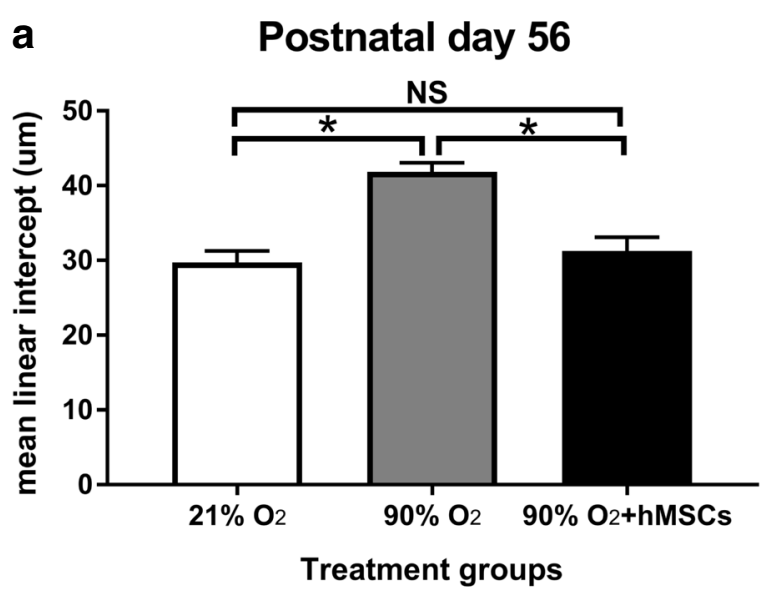

b

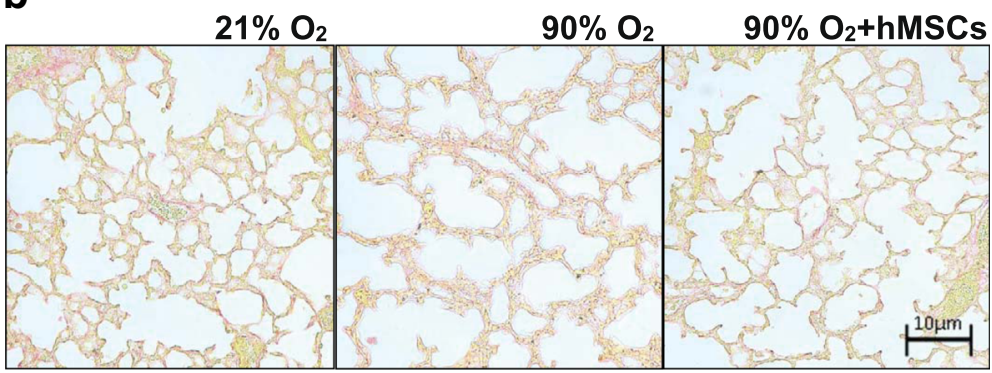

Fig. 9 a MLI following normoxia, $90 \% \mathrm{O}_{2}(\square)$ and $90 \% \mathrm{O}_{2}+$ hMSC $\left(\square .90 \% \mathrm{O}_{2}\right.$ was given between postnatal days $0-4$ and MSCs were given on postnatal day 4. For all treatment groups at day 56 ( $n=8$ per group). Data were analysed using a one-way ANOVA and shown as mean \pm SEM. $p<0.05$. $\mathbf{b}$ representative histological images of the three treatment groups stained with picrosirius red

The exposure of neonatal mice to $90 \% \mathrm{O}_{2}$ induced lung injury by postnatal day 14 , where there was an accumulation of interstitial collagen which is consistent with a previous report [32]. This finding was associated with pathological changes to the lungs, namely alveolar wall thickening and pathological changes indicative of emphysema [30].

The current study used polychromatic flow cytometry analysis to identify and compare granulocytes and macrophage phenotypes in the normoxic lung to the inflammatory lung following hyperoxic injury.

Our hypothesis on the lung response to hyperoxic injury was assessed by quantifying the inflammatory cells, a method we could study by using the flow cytometric assay.

In this study, $\mathrm{CD} 45^{+}$leukocytes were used to quantify the two subsets of granulocytes and macrophages without reference to the other leukocyte subsets (natural killer cells, invariant natural killer T-cell, T-helper cell, Cytotoxic T-cell, Dendritic cell, monocytes) [33].

The use of separate markers against Ly6C and Ly6G allowed a defined demarcation of granulocytes from other CD45 myeloid cells populations [34]. Furthermore, we used the standard approach of staining with both $\mathrm{CD} 11 \mathrm{~b}$ and $\mathrm{CD} 11 \mathrm{c}$ markers to differentiate macrophages from other myeloid cell populations [33, 34]. The increased proportion of granulocytes indicated an inflammatory response following four days of exposure to hyperoxia, which was consistent with studies showing that granulocytes are the predominant cell type that infiltrates the lung tissue following an injury $[35,36]$. The inflammatory environment may provide important cues leading to the infiltration of other inflammatory cells, including blood monocytes, that have the propensity to differentiate into M1 and M2 macrophages [37].

We have shown that hMSC attenuates the increase in the total number of $\mathrm{CD} 45^{+}$leukocyte $(P<0.05)$ at day 7 in the neonatal lung following 4 days of exposure to hyperoxia. The elevation of the leukocytes occurred as a result of primary granulocytes recruitment into the alveolar spaces and pulmonary interstitial parenchyma, as defined previously in different lung injury literature [38].

In the present study, the cell count of $\mathrm{Ly}_{6} \mathrm{C}^{+} \mathrm{Ly} 6 \mathrm{G}^{+}$ granulocytes in lung tissue was elevated at day 4 in the mice exposed to hyperoxia and reduced thereafter. Moreover, the administration of hMSCs was shown to have no effect of granulocyte number in hyperoxic lungs at day 7 and day 14. However, other studies suggest that the granulocytes might raise as a result of releasing granulocyte colony stimulating factor from the stem cells [39]. 
This study showed that the CD11b CD11c expressed macrophages were relatively high from day zero, although the level of CD11c expression was low in comparison with day 4 and 7 as shown in a representative FACS plots in Fig. 2. It is hard to confirm whether these macrophages are monocytes derived macrophages or foetal monocytesmacrophages, and immature-macrophages, with significant overlap in expression of marker sets. These interstitial macrophages were derived from the yolk sac [12] which initially derived from mesoderm cells that develop during gastrulation from the primitive streak [40].

Throughout the study, we demonstrated that CD11b ${ }^{+}$ $\mathrm{CD}_{11 \mathrm{c}^{+}}$macrophages increased in response to hyperoxia by day 14 , including an increased population of $\mathrm{F} 4 / 80^{\text {low }} \mathrm{CD} 206{ }^{\text {low }}$ inflammatory 'M1' macrophages and decreased $\mathrm{F} 4 / 80^{\text {high }} \mathrm{CD} 206^{\text {high }}$ anti-inflammatory ' $\mathrm{M} 2$ ' macrophages. In other studies, M1 macrophage infiltration has been confirmed as well, together with myeloid differentiation and the alteration of relative function at the site of inflammation [41, 42]. These changes in phenotype result in the activation of monocytes at different maturation stages leading to mature macrophages of distinctive functional states [43]. Because macrophages are necessary for the phagocytosis of apoptotic neutrophils, the exposure to neonatal hyperoxia may lead to a reduced number of macrophages proceeding to necrosis, leading to the expansion of damaged alveoli [11]. This conclusion contradicts studies suggesting that macrophages might secrete certain chemokines, which could influence neutrophil infiltration and recruitment [11].

Our data confirms the beneficial effect of hMSC treatment on the phenotype and function of macrophages after neonatal hyperoxia. The administration of MSCs to mice with hyperoxia-induced acute lung injury was shown alteration of the macrophages proportion and phenotypes. These findings indicated the presence of an inflammatory environment. These results have also been demonstrated in an experimental model of asthma after bone marrowderived hMSC administration [44].

Following the intra-airway administration of hMSCs, an increase in M2 macrophages was observed in the injured lungs of mice. It is possible that the reduction in lung injury in hMSC-treated mice was due to downregulation of proinflammatory factors and the upregulation of anti-inflammatory chemokines. In contrast, the advantageous effects of hMSC treatment were displayed by reducing eosinophil infiltration in mouse models with allergic lung injury [45]. These findings and other supporting studies suggested that hMSCs are able to alter the balance of macrophage phenotype and function that occur during injury to promote repair [46]. We showed that the protective effect of hMSCs in hyperoxia-induced lung injury could alter macrophage phenotype. Recent studies have shown the in vivo interaction between
hMSCs and macrophages to promote M2 polarisation. The in vitro co-culture of hMSCs and macrophages resulted in an alternatively activated macrophage phenotype characterised as mannose receptor (MR) high, IL-10high, IL-6high, TNF- $\alpha$ low and IL-12low which exhibits enhanced phagocytic activity, increased secretion of IL-10 and VEGF and decreased secretion of pro-inflammatory cytokines [44]. Further investigation to identify the polarisation mechanism will be important for the understanding how hMSCs alter the host response following therapeutic delivery, in the context not only the inflammatory response to hyperoxia, but also lung disorders such as allergic asthma and COPD. Apart from the alteration of lung myeloid cells, lung fibrosis is the final common pathway of lung injury regardless of aetiology. In examining the effect of hMSCs therapy on lung injury induced by $90 \% \mathrm{O}_{2}$, we chose an end-point of delivery of oxygen of four days as published data has shown that delivery of hMSCs may ameliorate established lung fibrosis after 14 days of hyperoxia- induced injury [47]. We found that the treatment with hMSCs induced decreased lung collagen accumulation following hyperoxia. We propose that hMSCs are able to create a more favourable environment, leading to less tissue damage and fibrosis. In this environment, hMSCs may possibly not only alter lung immune cells but may also have an increased capacity to facilitate their antifibrotic and reparative effects, resulting in a greater reduction in fibrosis [48]. The administration of hMSCs to the neonatal lung ameliorated the hyperoxia-induced structural injuries, including reducing collagen deposition. Additionally, the intratracheal delivery of hMSC was found to effectively reduce the hyperoxia-induced infiltration of myeloid cells, including sub-populations of macrophages, into the damaged lung tissue. This indicates that hMSCs can modulate the inflammatory environment in the lung to reduce the development of fibrotic damage and structural injury.

Using hMSCs in a wide range of settings has shown impressive treatment responses as these cells release anti-inflammatory factors providing a positive effect by modulating the inflammatory environments, hence improving tissue healing [46]. Our study has shown the effect of the administration of hMSCs immediately, after exposure to hyperoxia, on myeloid cell sub-populations in the lung. Given our findings, it is possible that future clinical trials may incorporate the use of hMSCs therapy to reduce the lung injury including injury that may developed following in preterm birth hyperoxia and other lung diseases that results from pathologic fibrosis. This study was designed to test the efficacy of hMSCs in limiting hyperoxia-induced lung injury. In order to establish a 'proof of principle' we used 90\% oxygen, which is a higher concentration of oxygen that is used clinically. It is recognised that our hyperoxic lung injury 
mouse model is likely to induce a more severe form of BPD than in preterm infants in normal physiological condition. In the future, it will be critical to assess the effectiveness of hMSC in lung injury models that more closely replicate clinical conditions. Additionally, the exposure to $90 \% \mathrm{O}_{2}$ is likely to cause hyperoxic injury in organs other than the lungs. As hMSCs administered intratracheally will likely home to injured tissues, it will be important study the systemic effects of hMSC in future studies.

\section{Conclusions}

Our study provides evidence for a novel cellular-based therapy that can induce lung repair and limit the progression of fibrosis in neonatal lungs exposed to hyperoxic gas. The results from this study demonstrate that hMSC therapy may provide improved therapeutic options for preterm infants necessarily exposed to hyperoxic gas, and may therefore reduce the risk of COPD and asthma in later life.

\section{Additional file}

Additional file 1: Table S1. Table showing the most differentially expressed genes in isolated macrophages from lungs of mice $(n=9)$ exposed to normoxia or hyperoxia with/without administration of hMSCs. RNA was isolated, then analysed, using next generation sequencing. The genes that are related to $\mathrm{M} 1$ and $\mathrm{M} 2$ macrophages were either up-regulated or down-regulated in isolated lung macrophages in response to hyperoxia, that were altered following delivery of hMSCs. Abbreviations: Cd86 (Cluster of Differentiation 86), Socs3 (suppressor of and cytokine signalling), Slamf1 (signalling lymphocytic activation molecule family member), Tnf (tumour necrosis factor), Fcgr1 (Fc receptor, lgG, low affinity), II12b (Interleukin 12B), II1b (Interleukin 1 beta), I16 (Interleukin 6), Arg1 (arginase enzyme gene), Stat6 (Signal transducer and activator of transcription 6) Retnla (resistin like alpha), Mrc1 (Mannose Receptor C-Type 1), II27ra (Interleukin 27 Receptor Subunit Alpha), II12b (Interleukin 12B). Data showing the Log2-Fold change in gene expression between the treatments groups (false discovery rate FDR $P$-value $<0.05$ ) for each expression. (PDF $16 \mathrm{~kb}$ )

\section{Abbreviations}

BPD: Bronchopulmonary dysplasia; COPD: Chronic Obstructive Pulmonary Disease; eGFP: Enhanced Green Fluorescent Protein; FACS: Fluorescenceactivated cell sorting; FMO: Fluorescence minus one; HBSS: Hanks' Balanced Salt Solution.; hMSCs: Human mesenchymal stem cells; MLI: Mean linear intercept; NGS: Next generation sequencing; SEM: Standard error of the mean

\section{Acknowledgments}

The authors acknowledge technical support from the staff at the Monash Histology Platform, Monash University and Monash Animal Research Platform, Core flow cytometry facility and Monash Bioinformatics Platform.

\section{Funding}

S R. is supported by Faculty of Medicine Nursing and Health Science, Monash University. C S. is supported by a National Health \&amp; Medical Research Council (NHMRC) of Australia Senior Research Fellowship (GNT1041766). A A. is supported by College of Medicine, Al-Nahrain University, Baghdad, Iraq.

\section{Availability of data and materials}

The datasets used and/or analysed during the current study are available from the corresponding author on reasonable request.

\section{Authors' contributions}

$\mathrm{AA}, \mathrm{SR}$ and $\mathrm{RH}$ designed the research; AA performed experiments and analysed data; AA and AW cultured human mesenchymal stem cells; AA and CS performed the hydroxyproline assay; AA wrote the paper with edits from all authors; $\mathrm{SR}, \mathrm{RH}$, FS and RDM intellectually contributed to project design and interpretation. All authors read and approved the final manuscript.

\section{Ethics approval}

The study was conducted under animal ethics number MARP2014/092 issued by Monash Animal Ethics Committee.

\section{Competing interests}

The authors declare that they have no competing interests.

\section{Publisher's Note}

Springer Nature remains neutral with regard to jurisdictional claims in published maps and institutional affiliations.

\section{Author details}

${ }^{1}$ Department of Anatomy and Developmental Biology, Biomedicine Discovery Institute, Monash University, Clayton, VIC 3800, Australia. ${ }^{2}$ Department of Pharmacology, Biomedicine Discovery Institute, Monash University, Clayton, Australia.

Received: 17 January 2018 Accepted: 21 May 2018

Published online: 08 June 2018

\section{References}

1. O'Reilly M, Sozo F, Harding R. Impact of preterm birth and bronchopulmonary dysplasia on the developing lung: long-term consequences for respiratory health. Clin Exp Pharmacol Physiol. 2013;40(11):765-73.

2. Stockwell S. Benefits of kangaroo Care for Premature Babies Continue into young adulthood. Am J Nurs. 2017:117(3):15.

3. Maitre NL, et al. Respiratory consequences of prematurity: evolution of a diagnosis and development of a comprehensive approach. J Perinatol. 2015;35(5):313-21.

4. Iyer NP, Mhanna MJ. The role of surfactant and non-invasive mechanical ventilation in early management of respiratory distress syndrome in premature infants. World J Pediatr. 2014;10(3):204-10.

5. Malkar MB, et al. Respiratory severity score on day of life 30 is predictive of mortality and the length of mechanical ventilation in premature infants with protracted ventilation. Pediatr Pulmonol. 2015;50(4):363-9.

6. Groneck P, et al. Association of pulmonary inflammation and increased microvascular permeability during the development of bronchopulmonary dysplasia: a sequential analysis of inflammatory mediators in respiratory fluids of high-risk preterm neonates. Pediatrics. 1994:93(5):712-8.

7. Voltolini $C$, et al. Understanding spontaneous preterm birth: from underlying mechanisms to predictive and preventive interventions. Reprod Sci. 2013;20(11):1274-92.

8. Bancalari E, Jain D, Jobe AH. Prevention of bronchopulmonary dysplasia: are Intratracheal steroids with surfactant a magic bullet? Am J Respir Crit Care Med. 2016;193(1):12-3.

9. Doyle LW, et al. Bronchopulmonary dysplasia in very low birth weight subjects and lung function in late adolescence. Pediatrics. 2006;118(1):108-13.

10. Xu YP. Bronchopulmonary dysplasia in preterm infants born at less than 32 weeks gestation. Glob Pediatr Health. 2016;3:2333794X16668773.

11. Vozzelli MA, et al. Antimacrophage chemokine treatment prevents neutrophil and macrophage influx in hyperoxia-exposed newborn rat lung. Am J Physiol Lung Cell Mol Physiol. 2004:286(3):L488-93.

12. Guilliams M, et al. Alveolar macrophages develop from fetal monocytes that differentiate into long-lived cells in the first week of life via GM-CSF. J Exp Med. 2013;210(10):1977-92.

13. Jones CV, Ricardo SD. Macrophages and CSF-1: implications for development and beyond. Organogenesis. 2013;9(4):249-60.

14. Jones $\mathrm{CV}$, et al. M2 macrophage polarisation is associated with alveolar formation during postnatal lung development. Respir Res. 2013;14:41.

15. Cho DI, et al. Mesenchymal stem cells reciprocally regulate the M1/M2 balance in mouse bone marrow-derived macrophages. Exp Mol Med. 2014;46:e70

16. Wei $X$, et al. Mesenchymal stem cells: a new trend for cell therapy. Acta Pharmacol Sin. 2013;34(6):747-54. 
17. Cheng SL, Lin CH, Yao CL. Mesenchymal stem cell Administration in Patients with chronic obstructive pulmonary disease: state of the science. Stem Cells Int. 2017;2017:8916570.

18. Sdrimas K, Kourembanas S. MSC microvesicles for the treatment of lung disease: a new paradigm for cell-free therapy. Antioxid Redox Signal. 2014;21(13):1905-15.

19. van Haaften T, et al. Airway delivery of mesenchymal stem cells prevents arrested alveolar growth in neonatal lung injury in rats. Am J Respir Crit Care Med. 2009;180(11):1131-42.

20. Li Y, et al. Hypoxia promotes the skewed differentiation of umbilical cord mesenchymal stem cells toward type II alveolar epithelial cells by regulating microRNA-145. Gene. 2017;630:68-75.

21. Aslam $\mathrm{M}$, et al. Bone marrow stromal cells attenuate lung injury in a murine model of neonatal chronic lung disease. Am J Respir Crit Care Med. 2009;180(11):1122-30.

22. Akyurekli $C$, et al. A systematic review of preclinical studies on the therapeutic potential of mesenchymal stromal cell-derived microvesicles. Stem Cell Rev. 2015;11(1):150-60.

23. Huuskes BM, et al. Combination therapy of mesenchymal stem cells and serelaxin effectively attenuates renal fibrosis in obstructive nephropathy. FASEB J. 2015:29(2):540-53.

24. Law CW, et al. Voom: precision weights unlock linear model analysis tools for RNA-seq read counts. Genome Biol. 2014;15(2):R29.

25. Ritchie ME, et al. Limma powers differential expression analyses for RNAsequencing and microarray studies. Nucleic Acids Res. 2015;43(7):e47.

26. Gallop PM, Paz MA. Posttranslational protein modifications, with special attention to collagen and elastin. Physiol Rev. 1975;55(3):418-87.

27. O'Reilly $\mathrm{M}$, et al. Bronchiolar remodeling in adult mice following neonatal exposure to hyperoxia: relation to growth. Anat Rec (Hoboken). 2014;297(4): 758-69.

28. Togel $\mathrm{F}$, et al. Bioluminescence imaging to monitor the in vivo distribution of administered mesenchymal stem cells in acute kidney injury. Am J Physiol Renal Physiol. 2008;295(1):F315-21

29. Gensel JC, et al. Predictive screening of $M 1$ and $M 2$ macrophages reveals the immunomodulatory effectiveness of post spinal cord injury azithromycin treatment. Sci Rep. 2017;7:40144

30. Sozo F, et al. Altered lung function at mid-adulthood in mice following neonatal exposure to hyperoxia. Respir Physiol Neurobiol. 2015:218:21-7.

31. Liu YY, et al. Hypoxia-preconditioned mesenchymal stem cells ameliorate ischemia/reperfusion-induced lung injury. PLoS One. 2017;12(11):e0187637.

32. Mascaretti RS, et al. Lung morphometry, collagen and elastin content: changes after hyperoxic exposure in preterm rabbits. Clinics (Sao Paulo). 2009;64(11):1099-104.

33. Misharin AV, et al. Flow cytometric analysis of macrophages and dendritic cell subsets in the mouse lung. Am J Respir Cell Mol Biol. 2013:49(4):503-10.

34. Rose S, Misharin A, Perlman H. A novel Ly6C/Ly6G-based strategy to analyze the mouse splenic myeloid compartment. Cytometry A. 2012;81(4):343-50.

35. Ohnishi T, et al. Mature hepatocyte growth factor/scatter factor on the surface of human granulocytes is released by a mechanism involving activated factor Xa. J Immunol. 2006;176(11):6945-53.

36. Sarangi PP, et al. Role of beta1 integrin in tissue homing of neutrophils during sepsis. Shock. 2012;38(3):281-7.

37. Cuenca J, et al. Infiltration of inflammatory cells plays an important role in matrix metalloproteinase expression and activation in the heart during sepsis. Am J Pathol. 2006;169(5):1567-76.

38. Reutershan J, et al. Sequential recruitment of neutrophils into lung and bronchoalveolar lavage fluid in LPS-induced acute lung injury. Am J Phys Lung Cell Mol Phys. 2005;289(5):L807-15.

39. Semedo $P$, et al. Bone marrow mononuclear cells attenuate fibrosis development after severe acute kidney injury. Lab Investig. 2010;90(5):685-95.

40. Palis J. Interaction of the macrophage and primitive erythroid lineages in the mammalian embryo. Front Immunol. 2016;7:669.

41. Giamarellos-Bourboulis EJ, Raftogiannis M. The immune response to severe bacterial infections: consequences for therapy. Expert Rev Anti-Infect Ther. 2012;10(3):369-80

42. Pillay J, et al. A subset of neutrophils in human systemic inflammation inhibits T cell responses through mac-1. J Clin Invest. 2012;122(1):327-36.

43. Sunderkotter $C$, et al. Subpopulations of mouse blood monocytes differ in maturation stage and inflammatory response. J Immunol. 2004;172(7):4410-7.

44. Mathias $\amalg$, et al. Alveolar macrophages are critical for the inhibition of allergic asthma by mesenchymal stromal cells. J Immunol. 2013;191(12):5914-24.
45. de Castro LL, et al. Human adipose tissue mesenchymal stromal cells and their extracellular vesicles act differentially on lung mechanics and inflammation in experimental allergic asthma. Stem Cell Res Ther. 2017;8(1):151.

46. Ionescu $L$, et al. Stem cell conditioned medium improves acute lung injury in mice: in vivo evidence for stem cell paracrine action. Am J Physiol Lung Cell Mol Physiol. 2012;303(11):L967-77.

47. Hansmann $\mathrm{G}$, et al. Mesenchymal stem cell-mediated reversal of bronchopulmonary dysplasia and associated pulmonary hypertension. Pulm Circ. 2012;2(2):170-81.

48. Adhyatmika A, et al. The elusive Antifibrotic macrophage. Front Med (Lausanne). 2015:2:81.

\section{Ready to submit your research? Choose BMC and benefit from:}

- fast, convenient online submission

- thorough peer review by experienced researchers in your field

- rapid publication on acceptance

- support for research data, including large and complex data types

- gold Open Access which fosters wider collaboration and increased citations

- maximum visibility for your research: over $100 \mathrm{M}$ website views per year

At BMC, research is always in progress.

Learn more biomedcentral.com/submissions 